\title{
Towards a "canonical" agranular cortical microcircuit
}

\section{Sarah F. Beul ${ }^{1}$ * and Claus C. Hilgetag ${ }^{1,2}$}

${ }^{1}$ Department of Computational Neuroscience, University Medical Center Hamburg-Eppendorf, Hamburg, Germany

${ }^{2}$ Department of Health Sciences, Boston University, Boston, MA, USA

Edited by:

Patrik Krieger, Ruhr University

Bochum, Germany

Reviewed by:

Giorgio Innocenti, Karolinska

Institutet, Sweden

Kathleen S. Rockland, Boston

University School Medicine, USA

\section{*Correspondence:}

Sarah F. Beul, Department of

Computational Neuroscience, University Medical Center

Hamburg-Eppendorf, Martinistrasse

52, 20246 Hamburg, Germany

e-mail: s.beul@uke.de
Based on regularities in the intrinsic microcircuitry of cortical areas, variants of a "canonical" cortical microcircuit have been proposed and widely adopted, particularly in computational neuroscience and neuroinformatics. However, this circuit is founded on striate cortex, which manifests perhaps the most extreme instance of cortical organization, in terms of a very high density of cells in highly differentiated cortical layers. Most other cortical regions have a less well differentiated architecture, stretching in gradients from the very dense eulaminate primary cortical areas to the other extreme of dysgranular and agranular areas of low density and poor laminar differentiation. It is unlikely for the patterns of inter- and intra-laminar connections to be uniform in spite of strong variations of their structural substrate. This assumption is corroborated by reports of divergence in intrinsic circuitry across the cortex. Consequently, it remains an important goal to define local microcircuits for a variety of cortical types, in particular, agranular cortical regions. As a counterpoint to the striate microcircuit, which may be anchored in an exceptional cytoarchitecture, we here outline a tentative microcircuit for agranular cortex. The circuit is based on a synthesis of the available literature on the local microcircuitry in agranular cortical areas of the rodent brain, investigated by anatomical and electrophysiological approaches. A central observation of these investigations is a weakening of interlaminar inhibition as cortical cytoarchitecture becomes less distinctive. Thus, our study of agranular microcircuitry revealed deviations from the well-known "canonical" microcircuit established for striate cortex, suggesting variations in the intrinsic circuitry across the cortex that may be functionally relevant.

Keywords: cytoarchitecture, intrinsic circuitry, interlaminar connectivity, striate cortex, structural variation

\section{INTRODUCTION}

The cerebral cortex is arguably one of the most complex physical systems. Untangling the intricate relations of the myriad elements of the gray matter is one of the formidable challenges of science, as already pronounced by Santiago Ramon y Cajal:

\footnotetext{
"Devotion to the cerebral hemispheres, enigma of enigmas, was old in me...the supreme cunning of the structure of the gray matter is so intricate that it defies and will continue to defy for many centuries the obstinate curiosity of investigators. That apparent disorder of the cerebral jungle, so different from the regularity and symmetry of the spinal cord and of the cerebellum, conceals a profound organization of the utmost subtlety which is at present inaccessible." (Cajal, 1937)
}

Decades later, the picture has become more refined, but a comprehensive understanding of the cortical microarchitecture still remains a fundamental scientific challenge. A crucial step was the recognition that the cerebral cortex is locally structured into horizontal compartments ("layers") as well as vertical units ("columns") which both may be of functional relevance. Traditionally, the isocortex has been characterized in the context of a six-layered scheme (Brodmann, 1909; Vogt, 1910; von Economo, 2009), as opposed to three-layered allocortex. This scheme is, however, subject to substantial variation in the relative prominence of layers and disrupted in a considerable number of cortical areas. Nonetheless, and in spite of his acknowledgment that "the distinction of six layers can be both arbitrary and conventional" (von Economo, 2009), already von Economo himself asserted that "on practical grounds, we retain the six-layer division" (von Economo, 2009). Indeed, the simplified concept of a uniformly six-layered isocortex has prevailed (Zilles and Amunts, 2012) and become generally accepted.

The radial organization of the cortex became a subject of interest when vertical columns spanning all cortical layers were proposed to exist (Lorente de Nó, 1949; Mountcastle, 1957), with uniform columns repeating across the cortex to form an intermediate-level neural substrate for information processing. Within these columns, connectivity across cortical layers appeared stereotypical (Szentagothai, 1978; Gilbert and Wiesel, 1983). While there is still considerable debate about the existence, precise definition and the extent of heterogeneity in the cellular composition of cortical columns (Rakic, 2008; da Costa and Martin, 2010; Rockland, 2010; Smith, 2010a,b,c,d; Carlo and Stevens, 2013; Herculano-Houzel et al., 2013), the concept of radial cortical organization was later extended to the notion of a "canonical" microcircuit (Douglas et al., 1989; Douglas and Martin, 1991, 

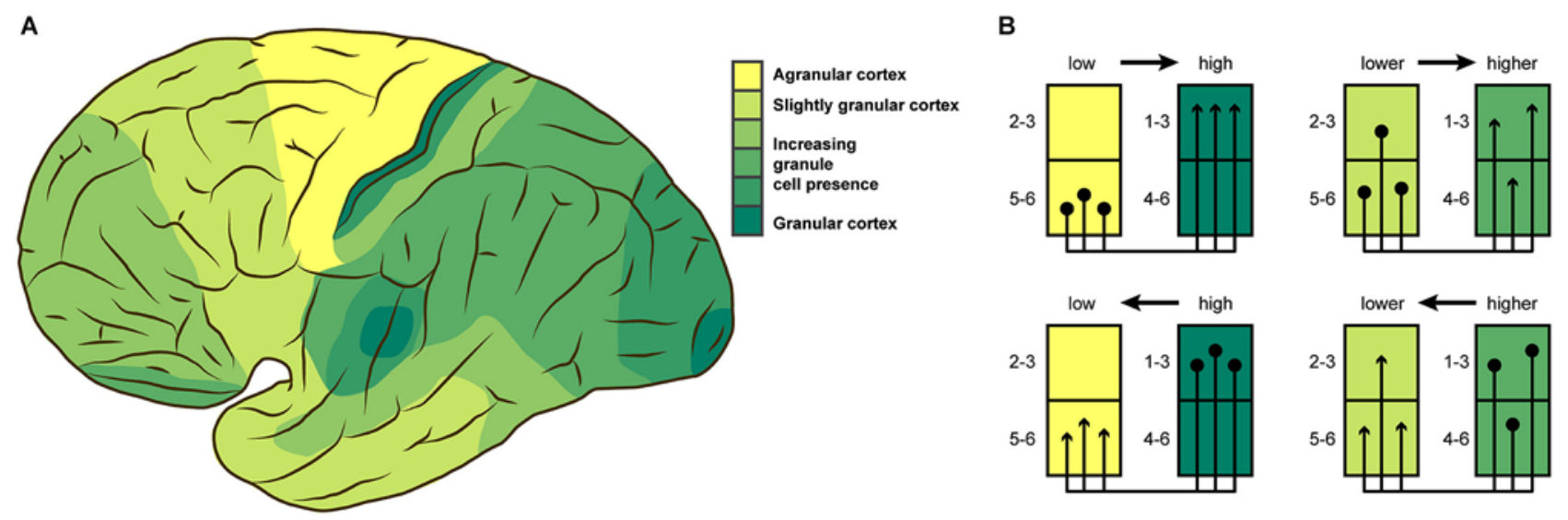

FIGURE 1 | (A) Cytoarchitectonic differentiation varies across the cortex. This lateral view of the human brain shows broad variations in granule cell presence as described by von Economo (2009). (B) Laminar origin and termination patterns of extrinsic cortico-cortical connections vary according to the relative architectonic differentiation of the connected areas. Projection origins (terminations) shift from infragranular to supragranular layers, as the source (target) area becomes more strongly differentiated. This rule results in unilaminar profiles for projections between areas that are unequal in their differentiation, and multilaminar profiles for areas with more similar differentiation. (A) adapted from von Economo (2009), (B) adapted from Barbas and Rempel-Clower (1997).
2004), as a generic template of intrinsic cortical circuitry. The computations performed by such a fundamental neuronal circuit are thought to be prescribed by the intrinsic circuitry within a cortical column, with functional specificity added by patterns of axonal inputs and outputs to and from the column. Substantial work has been devoted to the computational performance and theoretical properties of the "canonical" microcircuit (e.g., Douglas et al., 1989, 1995; Haeusler and Maass, 2007; George and Hawkins, 2009; Haeusler et al., 2009; Wagatsuma et al., 2011; Bastos et al., 2012; Habenschuss et al., 2013). In the primate prefrontal cortex, the "canonical" microcircuit was shown to be subject to modifications from the striate circuit (Heinzle et al., 2007; Godlove et al., 2014). More generally, abundant data is available on variants of intrinsic connectivity in cortical regions such as prefrontal cortex (Melchitzky et al., 2001), somatosensory cortex (Lübke and Feldmeyer, 2007; Petersen, 2007; Lefort et al., 2009; Feldmeyer et al., 2013) or auditory cortex (Barbour and Callaway, 2008; Oviedo et al., 2010; Watkins et al., 2014). Nonetheless, the notion of a "canonical" microcircuit, which has gained popularity especially in the computational neuroscience community and has also inspired neuroengineering solutions (e.g., Merolla et al., 2014), is still largely based on work in one particular cortical area, striate cortex. Moreover, much of this work has concentrated on the cat and non-human primate brain (Douglas and Martin, 2007a). Striate cortex is not only special in the amount of probing it has undergone, but is also exceptional in its cytoarchitectonic differentiation. Striate cortex is the cortical region with the highest neuron density, sporting numbers substantially higher than the remainder of the cortex (Schüz and Palm, 1989; Collins et al., 2010; Cahalane et al., 2012; Herculano-Houzel et al., 2013). The number of (sub)layers that can be identified is also higher in striate cortex than in other regions of the cortex. Instead of all parts of the cortex being uniformly differentiated, cytoarchitectonic differentiation changes gradually across the cortex (Sanides, 1970; von Economo, 2009; Zilles and Amunts, 2012), as illustrated in Figure 1A for the human brain. The spectrum of differentiation ranges from striate cortex, the most clearly eulaminate area, to agranular areas that lack the inner granular layer (L4), and have few identifiable sublayers as well as very low neuron density. In between these two extremes, one can find areas that are still eulaminate, but without the remarkable clarity of differentiation or dense packing of neurons found in striate cortex, such as prestriate cortex, as well as dysgranular areas with a lower density of neurons, a dissolving inner granular layer and fewer identifiable sublayers. Quantitative differences in many aspects of the structural organization of cortical tissue have been extensively demonstrated (e.g., Beaulieu and Colonnier, 1989; Defelipe et al., 1999; Dombrowski et al., 2001; Yáñez et al., 2005; Collins et al., 2010).

The variation in local cortical structure needs to be taken into account when describing a "canonical" microcircuit, because it is unlikely for the patterns of inter- and intra-laminar connections to be uniform in spite of strong variations of their structural substrate. Indeed, experimental results, for example from rodent barrel cortex, demonstrate that intrinsic connectivity is not uniform across the cortex (Sato et al., 2008; Meyer et al., 2013; Reyes-Puerta et al., 2014). Heterogeneity in cytoarchitectonic differentiation has been shown to have consequences for other aspects of structural connectivity in the brain. The laminar patterns of extrinsic connections which link brain regions along white matter pathways are strongly associated with the relative cytoarchitectonic differentiation of the connected areas (Barbas, 1986; Barbas and Rempel-Clower, 1997; Medalla and Barbas, 2006; Hilgetag and Grant, 2010; Beul et al., 2014). The stereotypic laminar patterns that have been found in non-human primate and cat cortex (Figure 1B) show distinctly infra- and supragranular origins and terminations for 
projections between areas of weak differentiation and areas of strong differentiation, while these patterns change gradually towards multilaminar origin and termination profiles as the difference in differentiation between the connected areas becomes less pronounced.

Since the variation of cytoarchitectonic differentiation is an aspect of cortical organization that is insufficiently considered in discussions of intrinsic circuitry, we here want to raise awareness of the importance of architectonic differences, by providing a first approximation of general features of intrinsic circuitry in agranular regions of the cerebral cortex. We do this by assimilating information from the available literature on inter- and intralaminar connectivity in the agranular frontal cortex of the rodent brain, in order to present a tentative model of intrinsic circuitry in cortical regions on the opposite end of the differentiation spectrum than has previously been predominantly considered for such models. This variation is crucial for applying insights gained from such model circuits in a realistic way, for example in the biological grounding of in silico experiments (e.g., Merolla et al., 2014).

In the following review, we briefly introduce current accounts of the "canonical" microcircuit, and then highlight a report of experimental results that reveal variation in interlaminar inhibition across cortical regions of distinct cytoarchitecture (Kätzel et al., 2011). Subsequently, we present the result of the literature survey we performed regarding data that can shed light on the intrinsic microcircuitry in agranular cortex. We chose to concentrate on the rodent brain, capitalizing on the relative abundance of experimental data available for this popular animal model. In comparison, fewer studies report on intrinsic circuitry in nonhuman primates, and only a small proportion of those considered agranular cortical regions, which are relatively infrequent in the primate brain. By focusing on the rodent brain, we can therefore provide a more detailed sketch of the intrinsic circuitry in agranular cortex without having to incorporate data across a wide range of species, which would have been a more uncertain approach.

\section{INTRINSIC CIRCUITRY IN GRANULAR CORTEX}

Over the last decades, general features of intrinsic circuitry in striate cortex have emerged from studies in the cat and nonhuman primate. Connections are proposed to form a processing loop across cortical layers, where recurrent excitation and inhibition are interlinked, which leads to amplification of inputs into the cortical column and appropriate modulation of the ensuing activity (Markram et al., 2004; Douglas and Martin, 2004, 2007a; Bannister, 2005; Lübke and Feldmeyer, 2007). To probe the local microcircuitry, diverse experimental methods with different degrees of sensitivity and reliability have been used. Two investigations that supplied the most comprehensive data on cat striate cortex employed electrophysiological and morphological approaches, respectively. Thomson et al. (2002) used dual intracellular recordings to characterize synaptic connections across cortical layers. Binzegger et al. (2004) reconstructed the morphology of neurons in striate cortex in three dimensions and estimated the number of synaptic contacts between different cell types. Both data sets have been adapted and used in various studies, for example, in the construction of computational models (e.g., Haeusler and Maass, 2007; Haeusler et al., 2009; Bastos et al., 2012; Du et al., 2012; Potjans and Diesmann, 2014). But even though the same model system, cat striate cortex, was considered across these studies, there currently exists no definite scheme of this area's intrinsic circuitry. There are, for example, diverging data on whether recurrent excitation occurs between L3 and L5 or between L4 and L3 (cf. Thomson et al., 2002; Thomson and Bannister, 2003 and Binzegger et al., 2004; Douglas and Martin, 2004).

Such discrepancies may be reconciled by future experimental findings. In contrast, reports of differences in interlaminar activation patterns across cortical regions point towards the existence of genuine variations in intrinsic circuitry across the brain. Kätzel et al. (2011) used genetically targeted photostimulation to comprehensively map inhibitory-to-excitatory connectivity in three distinct regions of mouse cortex. They assessed intra- and interlaminar connectivity in striate cortex, primary somatosensory and primary motor cortex. As mentioned before, striate cortex is by far the most differentiated cortical region, even in the rodent brain (HerculanoHouzel et al., 2013), where it is less well differentiated than for example in the primate. Primary somatosensory cortex, although still clearly eulaminate, is already much less dense and comprises fewer distinguishable sublayers, while primary motor cortex is even less cytoarchitectonically differentiated (Collins et al., 2010; Herculano-Houzel et al., 2013). Primary motor cortex thus ranges in the lower end of the differentiation spectrum with dysgranular cortical regions, although it is sometimes classified as agranular (lacking an inner granular layer, L4): see Shipp (2005) and García-Cabezas and Barbas (2014) for an extensive discussion of this issue. Other than probing connectivity in three cortical regions processing different modalities, this study can, therefore, be used to demonstrate potential differences regarding intrinsic circuitry in three areas occupying different positions in the differentiation spectrum. While Kätzel et al. (2011) report relatively uniform patterns of intralaminar inhibition across these three cortical regions, interlaminar inhibitory-to-excitatory connectivity differed substantially (Figure 2). In striate cortex, considerable interlaminar inhibition was observed between all layers (L2/3, L4, L5/6). In primary somatosensory cortex, a similar pattern of interlaminar inhibition was reported, but without inhibition between L2/3 and L5/6. In primary motor cortex, in contrast, no substantial inhibition between L2/3, L4, and L5/6 was evident. Thus, across the three sampled regions, interlaminar inhibitory-to-excitatory connectivity was progressively weaker in less cytoarchitectonically differentiated areas. Interpreting the results this way, we assume that they reflect genuine variation in the presence of interlaminar inhibition, and not the impact of other aspects of structural variation across the studied areas. For example, systematic differences in cellular morphology across the sampled areas could lead to skewed results from applying the same measurement approach to all areas. Nonetheless, these observations support the notion that intrinsic circuitry cannot be uniform in the face of considerable variation of the structural substrate, as is the case in regions of the cerebral cortex with profoundly differing cytoarchitectonic differentiation. 


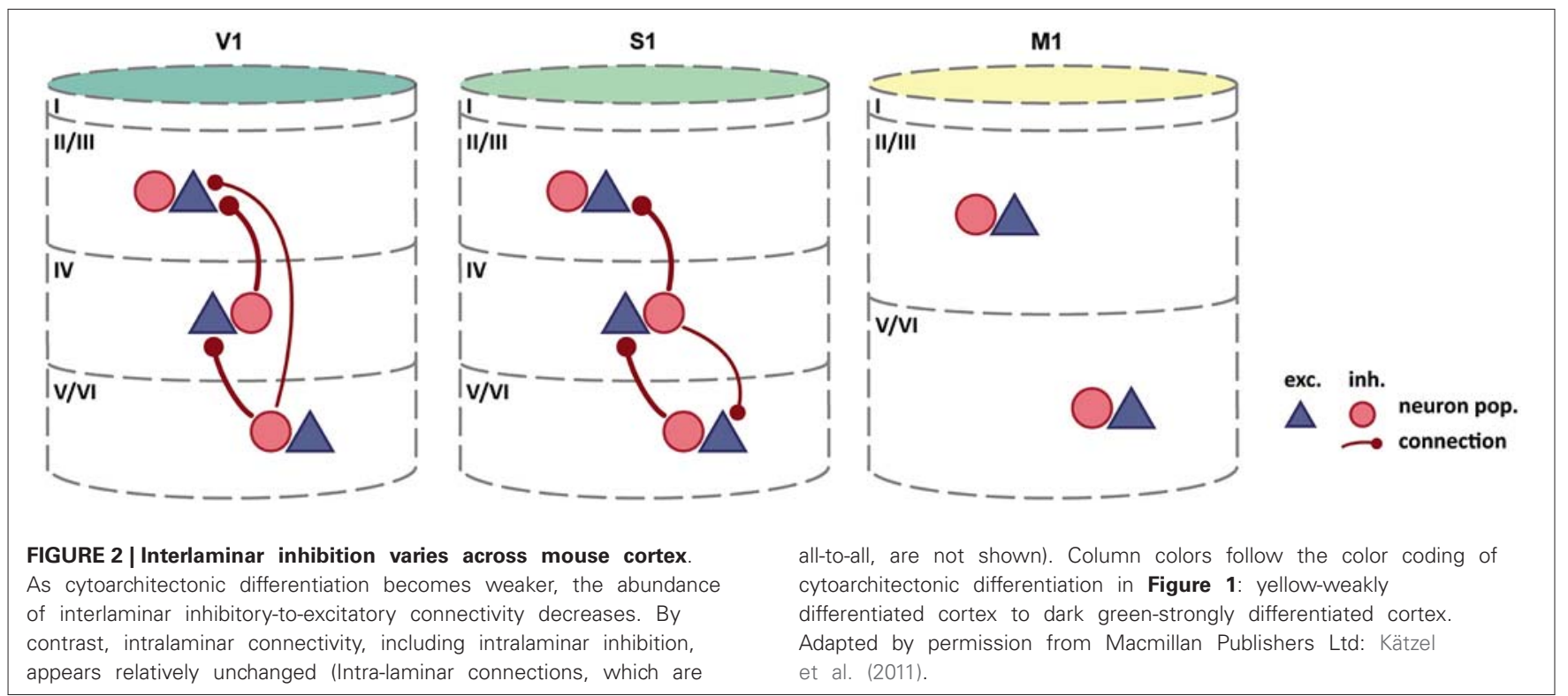

\section{TENTATIVE INTRINSIC CIRCUITRY OF THE AGRANULAR CORTEX}

Figure 3 summarizes our review of the available literature on intrinsic interlaminar circuitry in the agranular frontal cortex of the rodent brain and puts it in comparison to a recent rendering of the intrinsic circuitry in striate cortex. Excitatoryto-excitatory connections from L2/3 to L5 have clearly been demonstrated in rat agranular frontal cortex by measuring excitatory postsynaptic currents (EPSC) in monosynaptically coupled pyramidal neurons in L5, induced by stimulation in L2/3 (Kang, 1995; Otsuka and Kawaguchi, 2008, 2009, 2011; Hirai et al., 2012). One of these paired recording studies (Otsuka and Kawaguchi, 2009) additionally demonstrated the existence of excitatory-to-inhibitory connections from L2/3 to L5, a finding also reported by Apicella et al. (2012) in mouse motor cortex. The experiments of Hirai et al. (2012) showed that reciprocal connections to the excitatory-to-excitatory connections from L2/3 to L5 exist from L5 pyramidal cells to L2/3 pyramidal cells. This observation is confirmed in medial entorhinal cortex of the rat (van Haeften et al., 2003), which can be considered agranular since its layer IV ("lamina dissecans") is mainly acellular (Andersen et al., 2007). The microscopy study of van Haeften et al. (2003) traced the processes of pyramidal cells in the deep layers ramifying in superficial layers, and identified the synaptic contacts made by those neurons. The analysis revealed excitatory-to-excitatory, as well as excitatory-to-inhibitory, connections from deep to superficial layers.

Considering the trend of weakening inhibitory-to-excitatory connectivity in cytoarchitectonically less differentiated areas (Kätzel et al., 2011, see above), we consider it likely that there exists no substantial interlaminar inhibition of excitatory neurons in rodent agranular frontal cortex, which is reflected in our tentative circuit diagram. The study by van Haeften et al.
(2003) in medial entorhinal cortex, which reports an absence of inhibitory-to-excitatory synapses from deep to superficial layers, supports the same conclusion. Van Haeften et al. furthermore report that only a small percentage of the observed synapses could potentially be classified as inhibitory-to-inhibitory, thus giving little evidence for such a connection from deep to superficial layers. Considering the reciprocal inhibitory-to-inhibitory connection from superficial to deep layers, we could find no studies reporting either on the absence or presence of such a connection. In the circuit diagram (Figure 3), we did not include connections which could only be inferred from exclusively morphological results (e.g., Kawaguchi, 1993, 1995; Kawaguchi and Kubota, 1997; Kubota et al., 2011), since we did not consider data on the spatial spread of axon collaterals sufficiently reliable to demonstrate a functional connection, given that synapse formation has been shown to be highly specific (e.g., Kozloski et al., 2001; Brown and Hestrin, 2009). For these reasons, Figure 3B indicates no inhibitory interlaminar connections, although the validity of this assessment of course remains contingent upon further experimental data.

By contrast, there is abundant evidence for rich intralaminar connectivity including excitatory-to-inhibitory and inhibitoryto-excitatory connections (Kang, 1995; Somogyi et al., 1998; Kawaguchi and Kondo, 2002; Barthó et al., 2004; Otsuka and Kawaguchi, 2009; Fino and Yuste, 2011; Kätzel et al., 2011). Therefore, we assumed a stereotypical pattern of connectivity within deep and superficial layers as illustrated in Figure 3B.

The intrinsic circuitry we have sketched here thus comprises interlaminar excitatory connections that connect neuronal populations from both upper and lower layers to excitatory as well as inhibitory neuron populations in the complementary cortical layers. Within upper and lower layers, intralaminar connections reciprocally connect excitatory and inhibitory 


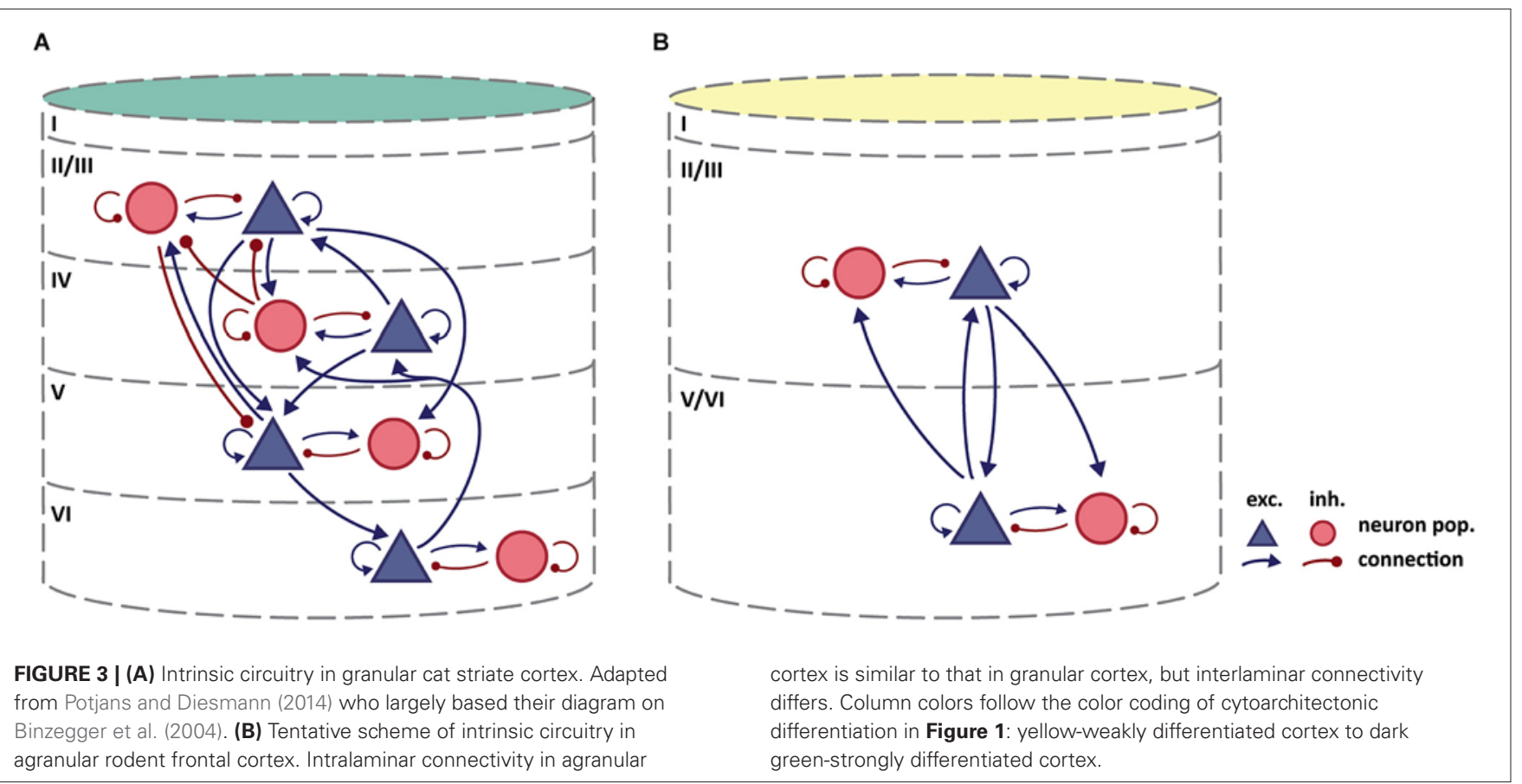

neuron populations. This intrinsic interlaminar circuitry is strikingly similar to the simplified original circuit diagram for the striate cortex of Douglas et al. (1989), and allows for recurrent excitation and inhibition. These physiological interactions were inferred to underlie essential computational mechanisms in striate cortex (Douglas et al., 1995; Douglas and Martin, 2007b, 2009). The microcircuitry as we sketch it here should accordingly be able to support elemental neural functions, such as the amplification of weak inputs through positive feedback or gain control and signal normalization through negative feedback.

\section{DISCUSSION}

The starting question of this review was whether there exists a generic template of intrinsic microcircuitry in the cortex, despite pronounced regional differences in cytoarchitectonic organization. The answer depends strongly on how broadly the concept of stereotypy is framed (Silberberg et al., 2002), but even for the cortical region studied most intensely in this context, striate cortex, there exists as yet no consensus on a detailed "canonical" microcircuit. Moreover, differences in circuitry have been reported across the cortex, which are consistent with the changes in the structural substrate in which intrinsic connectivity is embedded. In order to account for these structural differences, we propose a tentative circuit diagram for the agranular frontal cortex of the rodent brain, an agranular region which is strikingly opposed to striate cortex in its cytoarchitectonic organization. Our review of the existing literature points to an intrinsic circuit that features excitatory-to-excitatory and excitatory-to-inhibitory connections from upper layers to lower layers, as well as from lower layers to upper layers (Figure 3B), but shows no interlaminar inhibitoryto-inhibitory or inhibitory-to-excitatory connections. This circuit is based on multiple approaches for structural and functional circuit investigation (such as electrophysiological paired recordings using microstimulation, anatomical tracing experiments, or examination of morphological features using light and electron microscopy), with different caveats and varying levels of reliability. Importantly, the information was drawn from studies whose primary goal was not necessarily the characterization of interlaminar circuitry. Our circuit diagram is therefore subject to debate and should be modified in the light of future information. In compiling the circuit diagram, we engaged in some common simplifications regarding the anatomical substrate in which the connections are placed. In studying intrinsic circuitry, distinct sublayers are often collapsed, as for example when layers $5 \mathrm{~A}$, $5 \mathrm{~B}$ and 6 are considered collectively as "infragranular" layers. This treatment may be misleading, since different (sub)layers have been shown to be involved in distinct processing circuits (for example, Lübke and Feldmeyer, 2007). The same caveat holds for the merging of diverse neuron types into the two main classes of inhibitory and excitatory neurons. It discards a wealth of functionally relevant information about morphological and physiological differences between neuron types, as well as about cell type specific connectivity (Kozloski et al., 2001; Silberberg et al., 2002; Thomson and Bannister, 2003; Kampa et al., 2006; Otsuka and Kawaguchi, 2008, 2009, 2011; Brown and Hestrin, 2009; Xu and Callaway, 2009; Apicella et al., 2012; Hirai et al., 2012). Not to disambiguate such significant anatomical features introduces additional uncertainty about the validity of any intrinsic circuit diagram. Moreover, note that a description of general layer-to-layer connectivity within a column, as we propose here, does not necessarily reflect synaptic circuits formed by individual neurons across layers, as, for example, Binzegger et al. (2004) have estimated. Thus, there may exist functionally relevant differences 
between the average laminar interconnections described here and the specific laminar microcircuits formed within these average patterns. A further dimension that is missing from many descriptions of local microcircuitry is an estimation of connection strength. However, with current technology, structural measures of strength, such as the frequency of connections from one cell type onto another or the number of involved synapses and their morphology, can only be obtained by arduous manual labor. Moreover, the translation of structural into functional strength, as expressed by the amplitude of evoked postsynaptic currents, is opaque: number, size, morphology and position of synapses matter, as do numerous molecular mechanisms regulating synaptic function at both the pre- and postsynaptic site. In addition, the impact of evoked currents on postsynaptic cell function depends on many further factors. All these aspects are not static, but can potentially change on short time scales (Squire et al., 2008; Buonomano and Maass, 2009; Dityatev et al., 2010; Eroglu and Barres, 2010; Silver, 2010; Ribrault et al., 2011; Arnsten et al., 2012; Camiré and Topolnik, 2012; Caroni et al., 2012; CortésMendoza et al., 2013; Dallérac et al., 2013; Vitureira and Goda, 2013; Chevaleyre and Piskorowski, 2014).

Although the proposed intrinsic circuitry for agranular cortex is still speculative, the issue we address remains crucial (Marcus et al., 2014). There has to be variation in intrinsic circuitry across the cerebral cortex, because the composition of the cortex is not uniform, but highly variable on a number of dimensions. We are convinced that a better understanding of the intrinsic cortical circuitry is essential for an improved comprehension of its physiology, and has to take into account differences in the cortical structural substrate. We hope that we have provided a starting point for discussion which will lead to the synthetization of new insights from available data or further experimental efforts to elucidate circuitry outside of striate cortex, taking structural variation into consideration.

\section{ACKNOWLEDGMENTS}

Supported by DFG grant SFB 936/A1. We thank K.A.C. Martin anf $\mathrm{H}$. Barbas for helpful comments on the manuscript.

\section{REFERENCES}

Andersen, P., Morris, R., and Amaral, D. G. eds. (2007). The Hippocampus Book. New York: Oxford University Press.

Apicella, A. J., Wickersham, I. R., Seung, H. S., and Shepherd, G. M. G. (2012). Laminarly orthogonal excitation of fast-spiking and low-threshold-spiking interneurons in mouse motor cortex. J. Neurosci. 32, 7021-7033. doi: 10. 1523/JNEUROSCI.0011-12.2012

Arnsten, A. F. T., Wang, M. J., and Paspalas, C. D. (2012). Neuromodulation of thought: flexibilities and vulnerabilities in prefrontal cortical network synapses. Neuron 76, 223-239. doi: 10.1016/j.neuron.2012.08.038

Bannister, A. P. (2005). Inter- and intra-laminar connections of pyramidal cells in the neocortex. Neurosci. Res. 53, 95-103. doi: 10.1016/j.neures.2005.06.019

Barbas, H. (1986). Pattern in the laminar origin of corticocortical connections. J. Comp. Neurol. 252, 415-422. doi: 10.1002/cne.902520310

Barbas, H., and Rempel-Clower, N. L. (1997). Cortical structure predicts the pattern of corticocortical connections. Cereb. Cortex 7, 635-646. doi: 10. 1093/cercor/7.7.635

Barbour, D. L., and Callaway, E. M. (2008). Excitatory local connections of superficial neurons in rat auditory cortex. J. Neurosci. 28, 11174-11185. doi: 10. 1523/JNEUROSCI.2093-08.2008

Barthó, P., Hirase, H., Monconduit, L., Zugaro, M., Harris, K. D., and Buzsáki, G. (2004). Characterization of neocortical principal cells and interneurons by network interactions and extracellular features. J. Neurophysiol. 92, 600-608. doi: 10.1152/jn.01170.2003

Bastos, A. M., Usrey, W. M., Adams, R. A., Mangun, G. R., Fries, P., and Friston, K. J. (2012). Canonical microcircuits for predictive coding. Neuron 76, 695-711. doi: 10.1016/j.neuron.2012.10.038

Beaulieu, C., and Colonnier, M. (1989). Number of neurons in individual laminae of areas 3B, 4 gamma and $6 \mathrm{a}$ alpha of the cat cerebral cortex: a comparison with major visual areas. J. Comp. Neurol. 279, 228-234. doi: 10.1002/cne.9027 90206

Beul, S. F., Grant, S., and Hilgetag, C. C. (2014). A predictive model of the cat cortical connectome based on Cytoarchitecture and distance. Brain Struct. Funct. doi: 10.1007/s00429-014-0849-y. [Epub ahead of print].

Binzegger, T., Douglas, R. J., and Martin, K. A. C. (2004). A quantitative map of the circuit of cat primary visual cortex. J. Neurosci. 24, 8441-8453. doi: 10 . 1523/jneurosci.1400-04.2004

Brodmann, K. (1909). Vergleichende Lokalisationslehre der Großhirnrinde in ihren Prinzipien Dargestellt auf Grund des Zellenbaues. Leipzig: Johannes Ambrosius Barth Verlag.

Brown, S. P., and Hestrin, S. (2009). Intracortical circuits of pyramidal neurons reflect their long-range axonal targets. Nature 457, 1133-1136. doi: 10. 1038/nature 07658

Buonomano, D. V., and Maass, W. (2009). State-dependent computations: spatiotemporal processing in cortical networks. Nat. Rev. Neurosci. 10, 113-125. doi: $10.1038 / \mathrm{nrn} 2558$

Cahalane, D. J., Charvet, C. J., and Finlay, B. L. (2012). Systematic, balancing gradients in neuron density and number across the primate isocortex. Front. Neuroanat. 6:28. doi: 10.3389/fnana.2012.00028

Cajal, S. R. Y. (1937). Recollections of My Life. Philadelphia PA: American Philosophical Society.

Camiré, O., and Topolnik, L. (2012). Functional compartmentalisation and regulation of postsynaptic Ca2+ transients in inhibitory interneurons. Cell Calcium 52, 339-346. doi: 10.1016/j.ceca.2012.05.001

Carlo, C. N., and Stevens, C. F. (2013). Structural uniformity of neocortex, revisited. Proc. Natl. Acad. Sci. U S A 110, 1488-1493. doi: 10.1073/pnas.12213 98110

Caroni, P., Donato, F., and Muller, D. (2012). Structural plasticity upon learning: regulation and functions. Nat. Rev. Neurosci. 13, 478-490. doi: 10.1038/nrn 3258

Chevaleyre, V., and Piskorowski, R. A. (2014). Modulating excitation through plasticity at inhibitory synapses. Front. Cell. Neurosci. 8:93. doi: 10.3389/fncel. 2014.00093

Collins, C. E., Airey, D. C., Young, N. A., Leitch, D. B., and Kaas, J. H. (2010). Neuron densities vary across and within cortical areas in primates. Proc. Natl. Acad. Sci. U S A 107, 15927-15932. doi: 10.1073/pnas.1010356107

Cortés-Mendoza, J., Díaz de León-Guerrero, S., Pedraza-Alva, G., and PérezMartínez, L. (2013). Shaping synaptic plasticity: the role of activity-mediated epigenetic regulation on gene transcription. Int. J. Dev. Neurosci. 31, 359-369. doi: 10.1016/j.ijdevneu.2013.04.003

da Costa, N. M., and Martin, K. A. C. (2010). Whose cortical column would that be? Front. Neuroanat. 4:16. doi: 10.3389/fnana.2010.00016

Dallérac, G., Chever, O., and Rouach, N. (2013). How do astrocytes shape synaptic transmission? Insights from electrophysiology. Front. Cell. Neurosci. 7:159. doi: $10.3389 /$ fncel.2013.00159

Defelipe, J., González-Albo, M. C., Del Río, M. R., and Elston, G. N. (1999). Distribution and patterns of connectivity of interneurons containing calbindin, calretinin and parvalbumin in visual areas of the occipital and temporal lobes of the macaque monkey. J. Comp. Neurol. 412, 515-526. doi: 10.1002/(sici)10969861(19990927)412:3<515::aid-cne10>3.0.co;2-1

Dityatev, A., Schachner, M., and Sonderegger, P. (2010). The dual role of the extracellular matrix in synaptic plasticity and homeostasis. Nat. Rev. Neurosci. 11, 735-746. doi: 10.1038/nrn2898

Dombrowski, S. M., Hilgetag, C. C., and Barbas, H. (2001). Quantitative architecture distinguishes prefrontal cortical systems in the rhesus monkey. Cereb. Cortex 11, 975-988. doi: 10.1093/cercor/11.10.975

Douglas, R. J., Koch, C., Mahowald, M., Martin, K. A., and Suarez, H. H. (1995). Recurrent excitation in neocortical circuits. Science 269, 981-985. doi: 10. 1126/science.7638624

Douglas, R. J., and Martin, K. A. C. (1991). A functional microcircuit for cat visual cortex. J. Physiol. 440, 735-769. doi: 10.1113/jphysiol.1991.sp018733 
Douglas, R. J., and Martin, K. A. C. (2004). Neuronal circuits of the neocortex. Annu. Rev. Neurosci. 27, 419-451. doi: 10.1146/annurev.neuro.27.070203. 144152

Douglas, R. J., and Martin, K. A. C. (2007a). Recurrent neuronal circuits in the neocortex. Curr. Biol. 17, R496-R500. doi: 10.1016/j.cub.2007.04.024

Douglas, R. J., and Martin, K. A. C. (2007b). The butterfly and the loom. Brain Res. Rev. 55, 314-328. doi: 10.1016/j.brainresrev.2007.04.011

Douglas, R. J., and Martin, K. A. C. (2009). Inhibition in cortical circuits. Curr. Biol. 19, R398-R402. doi: 10.1016/j.cub.2009.03.003

Douglas, R. J., Martin, K. A. C., and Whitteridge, D. (1989). A canonical microcircuit for neocortex. Neural Comput. 1, 480-488. doi: 10.1162/neco.1989. 1.4.480

Du, J., Vegh, V., and Reutens, D. C. (2012). The laminar cortex model: a new continuum cortex model incorporating laminar architecture. PLoS Comput. Biol. 8:e1002733. doi: 10.1371/journal.pcbi.1002733

Eroglu, C., and Barres, B. A. (2010). Regulation of synaptic connectivity by glia. Nature 468, 223-231. doi: 10.1038/nature09612

Feldmeyer, D., Brecht, M., Helmchen, F., Petersen, C. C. H., Poulet, J. F. A., Staiger, J. F., et al. (2013). Barrel cortex function. Prog. Neurobiol. 103, 3-27. doi: 10. 1016/j.pneurobio.2012.11.002

Fino, E., and Yuste, R. (2011). Dense inhibitory connectivity in neocortex. Neuron 69, 1188-1203. doi: 10.1016/j.neuron.2011.02.025

García-Cabezas, M. Á., and Barbas, H. (2014). Area 4 has layer IV in adult primates. Eur. J. Neurosci. 39, 1824-1834. doi: 10.1111/ejn.12585

George, D., and Hawkins, J. (2009). Towards a mathematical theory of cortical micro-circuits. PLoS Comput. Biol. 5:e1000532. doi: 10.1371/journal.pcbi. 1000532

Gilbert, C. D., and Wiesel, T. N. (1983). Functional organization of the visual cortex. Prog. Brain Res. 58, 209-218. doi: 10.1016/S0079-6123(08)60022-9

Godlove, D. C., Maier, A., Woodman, G. F., and Schall, J. D. (2014). Microcircuitry of agranular frontal cortex: testing the generality of the canonical cortical microcircuit. J. Neurosci. 34, 5355-5369. doi: 10.1523/JNEUROSCI.5127-13. 2014

Habenschuss, S., Jonke, Z., and Maass, W. (2013). Stochastic computations in cortical microcircuit models. PLoS Comput. Biol. 9:e1003311. doi: 10.1371/journal. pcbi.1003311

Haeusler, S., and Maass, W. (2007). A statistical analysis of information-processing properties of lamina-specific cortical microcircuit models. Cereb. Cortex 17, 149-162. doi: 10.1093/cercor/bhj132

Haeusler, S., Schuch, K., and Maass, W. (2009). Motif distribution, dynamical properties and computational performance of two data-based cortical microcircuit templates. J. Physiol. Paris 103, 73-87. doi: 10.1016/j.jphysparis.2009.05.006

Heinzle, J., Hepp, K., and Martin, K. A. C. (2007). A microcircuit model of the frontal eye fields. J. Neurosci. 27, 9341-9353. doi: 10.1523/jneurosci.0974-07. 2007

Herculano-Houzel, S., Watson, C., and Paxinos, G. (2013). Distribution of neurons in functional areas of the mouse cerebral cortex reveals quantitatively different cortical zones. Front. Neuroanat. 7:35. doi: 10.3389/fnana.2013.00035

Hilgetag, C. C., and Grant, S. (2010). Cytoarchitectural differences are a key determinant of laminar projection origins in the visual cortex. Neuroimage 51, 1006-1017. doi: 10.1016/j.neuroimage.2010.03.006

Hirai, Y., Morishima, M., Karube, F., and Kawaguchi, Y. (2012). Specialized cortical subnetworks differentially connect frontal cortex to Parahippocampal areas. J. Neurosci. 32, 1898-1913. doi: 10.1523/JNEUROSCI.2810-11.2012

Kampa, B. M., Letzkus, J. J., and Stuart, G. J. (2006). Cortical feed-forward networks for binding different streams of sensory information. Nat. Neurosci. 9, 14721473. doi: $10.1038 / \mathrm{nn} 1798$

Kang, Y. (1995). Differential paired pulse depression of non-NMDA and NMDA currents in pyramidal cells of the rat frontal cortex. J. Neurosci. 15, 8268-8280.

Kätzel, D., Zemelman, B. V., Buetfering, C., Wölfel, M., and Miesenböck, G. (2011). The columnar and laminar organization of inhibitory connections to neocortical excitatory cells. Nat. Neurosci. 14, 100-107. doi: 10.1038/nn. 2687

Kawaguchi, Y. (1993). Groupings of nonpyramidal and pyramidal cells with specific physiological and morphological characteristics in rat frontal cortex. J. Neurophysiol. 69, 416-431.

Kawaguchi, Y. (1995). Physiological subgroups of nonpyramidal cells with specific morphological characteristics in layer II/III of rat frontal cortex. J. Neurosci. 15, 2638-2655.
Kawaguchi, Y., and Kondo, S. (2002). Parvalbumin, somatostatin and cholecystokinin as chemical markers for specific GABAergic interneuron types in the rat frontal cortex. J. Neurocytol. 31, 277-287. doi: 10.1023/A:1024126110356

Kawaguchi, Y., and Kubota, Y. (1997). GABAergic cell subtypes and their synaptic connections in rat frontal cortex. Cereb. Cortex 7, 476-486. doi: 10. 1093/cercor/7.6.476

Kozloski, J., Hamzei-Sichani, F., and Yuste, R. (2001). Stereotyped position of local synaptic targets in neocortex. Science 293, 868-872. doi: 10.1126/science.293. 5531.868

Kubota, Y., Shigematsu, N., Karube, F., Sekigawa, A., Kato, S., Yamaguchi, N., et al. (2011). Selective coexpression of multiple chemical markers defines discrete populations of neocortical GABAergic neurons. Cereb. Cortex 21, 1803-1817. doi: 10.1093/cercor/bhq252

Lefort, S., Tomm, C., Floyd Sarria, J.-C., and Petersen, C. C. H. (2009). The excitatory neuronal network of the $\mathrm{C} 2$ barrel column in mouse primary somatosensory cortex. Neuron 61, 301-316. doi: 10.1016/j.neuron. 2008.12.020

Lorente de Nó, R. (1949). "The cerebral cortex: architecture, intracortical connections, motor projections," in Physiology of the Nervous System, ed J. F. Fulton (Oxford: Oxford University Press), 288-330.

Lübke, J., and Feldmeyer, D. (2007). Excitatory signal flow and connectivity in a cortical column: focus on barrel cortex. Brain Struct. Funct. 212, 3-17. doi: 10 1007/s00429-007-0144-2

Marcus, G., Marblestone, A., and Dean, T. (2014). The atoms of neural computation. Science 346, 551-552. doi: 10.1126/science.1261661

Markram, H., Toledo-Rodriguez, M., Wang, Y., Gupta, A., Silberberg, G., and Wu, C. (2004). Interneurons of the neocortical inhibitory system. Nat. Rev. Neurosci. 5, 793-807. doi: 10.1038/nrn1519

Medalla, M., and Barbas, H. (2006). Diversity of laminar connections linking periarcuate and lateral intraparietal areas depends on cortical structure. Eur. J. Neurosci. 23, 161-179. doi: 10.1111/j.1460-9568.2005.04522.x

Melchitzky, D. S., González-Burgos, G., Barrionuevo, G., and Lewis, D. A. (2001). Synaptic targets of the intrinsic axon collaterals of supragranular pyramidal neurons in monkey prefrontal cortex. J. Comp. Neurol. 430, 209-221. doi: 10. 1002/1096-9861(20010205)430:2<209::aid-cne1026>3.0.co;2

Merolla, P. A., Arthur, J. V., Alvarez-Icaza, R., Cassidy, A. S., Sawada, J., Akopyan, F., et al. (2014). A million spiking-neuron integrated circuit with a scalable communication network and interface. Science 345, 668-673. doi: 10.1126/science. 1254642

Meyer, H. S., Egger, R., Guest, J. M., Foerster, R., Reissl, S., and Oberlaender, M. (2013). Cellular organization of cortical barrel columns is whisker-specific. Proc. Natl. Acad. Sci. U S A 110, 19113-19118. doi: 10.1073/pnas.13126 91110

Mountcastle, V. B. (1957). Modality and Topographic properties of single neurons of cat's somatic sensory cortex. J. Neurophysiol. 20, 408-434.

Otsuka, T., and Kawaguchi, Y. (2008). Firing-Pattern-Dependent specificity of cortical excitatory feed-forward subnetworks. J. Neurosci. 28, 11186-11195. doi: 10.1523/JNEUROSCI.1921-08.2008

Otsuka, T., and Kawaguchi, Y. (2009). Cortical inhibitory cell types differentially form Intralaminar and Interlaminar subnetworks with excitatory neurons. J. Neurosci. 29, 10533-10540. doi: 10.1523/JNEUROSCI.2219-09. 2009

Otsuka, T., and Kawaguchi, Y. (2011). Cell diversity and connection specificity between Callosal projection neurons in the frontal cortex. J. Neurosci. 31, 38623870. doi: 10.1523/JNEUROSCI.5795-10.2011

Oviedo, H. V., Bureau, I., Svoboda, K., and Zador, A. M. (2010). The functional asymmetry of auditory cortex is reflected in the organization of local cortical circuits. Nat. Neurosci. 13, 1413-1420. doi: 10.1038/nn.2659

Petersen, C. C. H. (2007). The functional organization of the barrel cortex. Neuron 56, 339-355. doi: 10.1016/j.neuron.2007.09.017

Potjans, T. C., and Diesmann, M. (2014). The cell-type specific cortical microcircuit: relating structure and activity in a full-scale spiking network model. Cereb. Cortex 24, 785-806. doi: 10.1093/cercor/bhs358

Rakic, P. (2008). Confusing cortical columns. Proc. Natl. Acad. Sci. U S A 105, 12099-12100. doi: 10.1073/pnas.0807271105

Reyes-Puerta, V., Sun, J.-J., Kim, S., Kilb, W., and Luhmann, H. J. (2014). Laminar and columnar structure of sensory-evoked multineuronal spike sequences in adult rat barrel cortex in vivo. Cereb. Cortex doi: 10.1093/cercor/bhu007. [Epub ahead of print]. 
Ribrault, C., Sekimoto, K., and Triller, A. (2011). From the stochasticity of molecular processes to the variability of synaptic transmission. Nat. Rev. Neurosci. 12, 375-387. doi: 10.1038/nrn3025

Rockland, K. S. (2010). Five points on columns. Front. Neuroanat. 4:22. doi: 10. 3389/fnana.2010.00022

Sanides, F. (1970). "Functional architecture of motor and sensory cortices in primates in the light of a new concept of neocortex evolution," in The Primate Brain, eds C. R. Noback and W. Montagna (New York: Appleton-CenturyCrofts), 137-208.

Sato, H., Shimanuki, Y., Saito, M., Toyoda, H., Nokubi, T., Maeda, Y., et al. (2008). Differential columnar processing in local circuits of barrel and insular cortices. J. Neurosci. 28, 3076-3089. doi: 10.1523/JNEUROSCI.0172-08.2008

Schüz, A., and Palm, G. (1989). Density of neurons and synapses in the cerebral cortex of the mouse. J. Comp. Neurol. 286, 442-455. doi: 10.1002/cne.9028 60404

Shipp, S. (2005). The importance of being agranular: a comparative account of visual and motor cortex. Philos. Trans. R. Soc. Lond. B Biol. Sci. 360, 797-814. doi: $10.1098 /$ rstb.2005.1630

Silberberg, G., Gupta, A., and Markram, H. (2002). Stereotypy in neocortical microcircuits. Trends Neurosci. 25, 227-230. doi: 10.1016/s0166-2236(02) 02151-3

Silver, R. A. (2010). Neuronal arithmetic. Nat. Rev. Neurosci. 11, 474-489. doi: 10. $1038 / \mathrm{nrn} 2864$

Smith, C. U. M. (2010a). Does history repeat itself? Cortical columns 1. Introduction. Cortex 46, 279-280. doi: 10.1016/j.cortex.2008.12.001

Smith, C. U. M. (2010b). Does history repeat itself? Cortical columns 2. From cytoarchitectonics to columns. Cortex 46, 591-592. doi: 10.1016/j.cortex.2008. 12.003

Smith, C. U. M. (2010c). Does history repeat itself? Cortical columns 3. A cortex of columns. Cortex 46, 713-714. doi: 10.1016/j.cortex.2008.12.002

Smith, C. U. M. (2010d). Does history repeat itself? Cortical columns 4. Déja vu? Cortex 46, 947-948. doi: 10.1016/j.cortex.2009.02.002

Somogyi, P., Tamás, G., Lujan, R., and Buhl, E. H. (1998). Salient features of synaptic organisation in the cerebral cortex. Brain Res. Rev. 26, 113-135. doi: 10. 1016/s0165-0173(97)00061-1

Squire, L. R., Berg, D., Bloom, F. E., du Lac, S., Ghosh, A., and Spitzer, N. eds. (2008). Fundamental Neuroscience. 3rd Edn. London: Elsevier Academic Press.

Szentagothai, J. (1978). The ferrier lecture, 1977: the neuron network of the cerebral cortex: a functional interpretation. Proc. R. Soc. Lond. B 201, 219-248. doi: 10. 1098/rspb.1978.0043

Thomson, A. M., and Bannister, A. P. (2003). Interlaminar connections in the neocortex. Cereb. Cortex 13, 5-14. doi: 10.1093/cercor/13.1.5

Thomson, A. M., West, D. C., Wang, Y., and Bannister, A. P. (2002). Synaptic connections and small circuits involving excitatory and inhibitory neurons in layers $2-5$ of adult rat and cat neocortex: triple intracellular recordings and biocytin labelling in vitro. Cereb. Cortex 12, 936-953. doi: 10.1093/cercor/ 12.9.936

van Haeften, T., Baks-te-Bulte, L., Goede, P. H., Wouterlood, F. G., and Witter, M. P. (2003). Morphological and numerical analysis of synaptic interactions between neurons in deep and superficial layers of the entorhinal cortex of the rat. Hippocampus 13, 943-952. doi: 10.1002/hipo.10144

Vitureira, N., and Goda, Y. (2013). The interplay between Hebbian and homeostatic synaptic plasticity. J. Cell Biol. 203, 175-186. doi: 10.1083/jcb.201306030

Vogt, O. (1910). Die myeloarchitektonische Felderung des menschlichen Stirnhirns. J. Psychol. Neurol. 15, 221-232.

von Economo, C. (2009). Cellular Structure of the Human Cerebral Cortex, ed L. C. Triarhou (Basel: Karger Medical and Scientific Publishers).

Wagatsuma, N., Potjans, T. C., Diesmann, M., and Fukai, T. (2011). Layerdependent attentional processing by top-down signals in a visual cortical microcircuit model. Front. Comput. Neurosci. 5:31. doi: 10.3389/fncom.2011. 00031

Watkins, P. V., Kao, J. P. Y., and Kanold, P. O. (2014). Spatial pattern of intra-laminar connectivity in supragranular mouse auditory cortex. Front. Neural. Circuits 8:15. doi: 10.3389/fncir.2014.00015

Xu, X., and Callaway, E. M. (2009). Laminar specificity of functional input to distinct types of inhibitory cortical neurons. J. Neurosci. 29, 70-85. doi: 10. 1523/JNEUROSCI.4104-08.2009

Yáñez, I. B., Muñoz, A., Contreras, J., Gonzalez, J., Rodriguez-Veiga, E., and DeFelipe, J. (2005). Double bouquet cell in the human cerebral cortex and a comparison with other mammals. J. Comp. Neurol. 486, 344-360. doi: 10. 1002/cne.20533

Zilles, K., and Amunts, K. (2012). "Architecture of the cerebral cortex," in The Human Nervous System, eds J. K. Mai and G. Paxinos (San Diego: Academic Press), 836-895. Available online at: http://www.sciencedirect.com/ science/article/pii/B9780123742360100239 Accessed on June 3, 2014.

Conflict of Interest Statement: The authors declare that the research was conducted in the absence of any commercial or financial relationships that could be construed as a potential conflict of interest.

Received: 15 July 2014; accepted: 19 December 2014; published online: 14 January 2015.

Citation: Beul SF and Hilgetag CC (2015) Towards a "canonical" agranular cortical microcircuit. Front. Neuroanat. 8:165. doi: 10.3389/fnana.2014.00165

This article was submitted to the journal Frontiers in Neuroanatomy.

Copyright (C) 2015 Beul and Hilgetag. This is an open-access article distributed under the terms of the Creative Commons Attribution License (CC BY). The use, distribution and reproduction in other forums is permitted, provided the original author(s) or licensor are credited and that the original publication in this journal is cited, in accordance with accepted academic practice. No use, distribution or reproduction is permitted which does not comply with these terms. 